\title{
O PESSEGUEIRO EM POMAR COMPACTO: X. COMPORTAMENTO DE CULTIVARES E SELEÇÕES SOB PODA DE ENCURTAMENTO DOS RAMOS PÓS-COLHEITA ${ }^{(1)}$
}

\author{
WILSON BARBOSA $^{(2,5)}$; FERNANDO ANTONIO CAMPO-DALL'ORTO ${ }^{(2,5)}$; \\ MÁRIO OJIMA ${ }^{(2,5)}$; MARIA DO CARMO DE SALVO SOARES NOVO ${ }^{(3)}$; \\ MARIA LUIZA CARVALHO CARELLI ${ }^{(3,5)}$; \\ JOAQUIM ADELINO DE AZEVEDO FILHO ${ }^{(4)}$

\section{RESUMO}

\begin{abstract}
Pesquisou-se o comportamento vegetativo e reprodutivo de 14 cultivares e seleções de pêssego e nectarina, cultivados no sistema de pomar compacto, em espaçamento de $4 \mathrm{~m} \mathrm{x} \mathrm{1,5} \mathrm{m}\left(1.667\right.$ plantas.ha $\left.^{-1}\right)$, sob poda de encurtamento dos ramos, em pós-colheita. Essa poda, caracterizada pelo corte dos ramos produtivos a 20-30 cm de sua inserção, foi realizada no final de novembro, logo após a colheita de frutos do ciclo anterior. A partir dos resultados obtidos no biênio 1997-98, verificou-se que os cultivares e seleções mais produtivos foram (média bienal do número e $\mathrm{kg}$ de frutos por planta respectivamente): Aurora-1 (161, 13,9); IAC 680-13 (142, 12,4); Régis (156, 12,2); Talismã $(128,11,9)$ e IAC 680-178 (130, 10,1). Os cultivares Delicioso Precoce, Jóia-1, Tropical, Flordaprince e IAC 2380-55, com produção intermediária, obtiveram índices entre 6,0 e $8,8 \mathrm{~kg}$ de frutos por planta. Durante os dois anos do experimento, as váriáveis fisiológicas analisadas - área de secção transversal do tronco, volume da copa, número e massa dos frutos por $\mathrm{cm}^{2}$ de tronco - apresentaram diferenciação estatística entre cultivares e seleções. A poda de encurtamento dos ramos, em pós-colheita, evitou a alternância de produção dos cultivares e seleções pesquisados.

Palavras-chave: pêssego, nectarina, Prunus persica, prática cultural, seleções IAC, volume de copa, área transversal de tronco, produtividade.
\end{abstract}

\section{ABSTRACT \\ THE PEACH MEADOW ORCHARD SYSTEM: X. BEHAVIOR OF CULTIVARS AND SELECTIONS ON SUMMER PRUNING AFTER HARVEST}

Eleven peach (Prunus persica L. Batsch) and three nectarine (P. persica L. Batsch, var. nucipersica) cultivars and selections, budded on Okinawa rootstocks, grown under the meadow orchard system at $4 \mathrm{~m} \times 1.5 \mathrm{~m}$ spacing, were summer pruned after harvest for two years. The experimental area was located in Monte Alegre do Sul (22 $41^{\prime}$ S; $46^{\circ} 43^{\prime}$ W) State of São Paulo, Brazil, with an average of chill accumulation of $40 \mathrm{~h}$ below $7^{\circ} \mathrm{C}$. From 1997 to 1998 cycles several physiological characteristics were recorded: trunk cross-sectional area, canopy volume, yield, fruits and mass per $\mathrm{cm}^{2}$ of trunk. The best

${ }^{1}$ ) Enviado para publicação em $1 .^{\circ}$ de julho de 1999 e aceito em 18 de julho de 2000.

$\left(^{2}\right)$ Centro de Fruticultura, Instituto Agronômico (IAC), Caixa Postal 28, 13001-970 Campinas (SP).

$\left({ }^{3}\right)$ Centro de Ecofisiologia e Biofísica, IAC.

$\left({ }_{5}^{4}\right)$ Estação Experimental de Agronomia de Monte Alegre do Sul, IAC, Caixa Postal 1, 13910-000 Monte Alegre do Sul (SP).

$\left(^{5}\right)$ Com bolsa de produtividade em pesquisa do CNPq. 
results were observed for Aurora-1, IAC 680-13, Régis, Talismã and IAC 680-178 cultivars and selections with an average of number of fruits and fruit yield $\left(\mathrm{kg}_{\text {.tree }}{ }^{-1}\right)$, respectively: 161, 13.9; 142, $12.4 ; 156,12.2 ; 128,11.9$ and $130,10.1$. The summer pruning increased the yield and medium fruit mass. Data on trunk cross-sectional area, canopy volume, yield and fruits and mass per $\mathrm{cm}^{2}$ of trunk presented significant differences among cultivars and selections.

Key words: peach, nectarine, Prunus persica, cultural practice, IAC selections, productivity, canopy volume, trunk cross-sectional area.

\section{INTRODUÇÃO}

O cultivo do pessegueiro, em pomar compacto, caracteriza-se pela redução máxima do espaçamento entre plantas e linhas e pelo controle do volume da copa, visando minimizar a competição negativa entre os indivíduos. Nas últimas duas décadas pesquisouse o plantio dessa frutífera em densidades populacionais de até cerca de 20.000 plantas por hectare, conduzidas em linhas únicas ou duplas, sob diferentes tipos de manejo cultural (GuERRIERO et al., 1980; CAMPO Dall'orto et al., 1984; EREZ, 1985; Loreti e PisAni, 1990; CARUSO et al., 1997; BARBOSA et al., 1989; 1998; 1999; SCARPARE FilHO et al., 1999).

Ao avanço maior desse sistema de cultivo do pessegueiro há que acrescentar, ainda, a necessidade de se controlar o excessivo vigor vegetativo das plantas. Várias pesquisas demonstraram que as podas drásticas anuais ou bienais podem reduzir satisfatoriamente o tamanho da copa, diminuindo a competição por energia solar, água, nutrientes e espaço físico (CAMPO-Dall'ORTO et al., 1984; Coston et al., 1985; EREZ, 1985; BARBOSA, 1989; BARBOSA et al., 1991; 1994). Observa-se, porém, que, se de um lado as podas drásticas proporcionam adequado balanceamento do desenvolvimento da copa, de outro lado, podem afetar as altas produtividades dos pomares. Assim sendo, a poda drástica anual só deve ser adotada para pessegueiros com características de maturação antecipada dos frutos. Empregando-se cultivares de ciclos precoces, há possibilidade de se podar mais cedo a copa - início da primavera - a fim de que ocorra rápida brotação do tronco decepado. Somente assim, os novos ramos reúnem condições ecofisiológicas para proporcionar a diferenciação floral das gemas, floração e maturação dos frutos, respectivamente, em cinco, nove, e doze meses de desenvolvimento (BARBOSA et al., 1990a). Através da poda drástica bienal pode-se viabilizar, para pomares compactos, o emprego de pessegueiros, tanto precoces como medianos e inclusive tardios. O fator desfavorável à bienalidade da poda drástica refere-se a certa alternância de produção dos cultivares não-precoces. Isso porque, em se realizando a poda drástica mais tarde - final da primavera - ocorre, obrigatoriamente, deficiência no desenvolvimento da nova copa e, portanto, na frutificação imediatamente posterior. No ciclo seguinte, com ausência programada da poda, a produtividade normal se restabelece, pois as copas se desenvolvem livremente durante todo ciclo vegetoreprodutivo. O Talismã, material mediano pesquisado durante dez anos em pomar compacto (1.667 plantas.ha $\left.{ }^{-1}\right)$, sob poda drástica bienal, constitui-se em exemplo bem ilustrativo. Esse cultivar produziu, em média, 6,0 kg.planta ${ }^{-1} \mathrm{em}$ anos submetidos à poda e, 10,2 kg.planta ${ }^{-1}$, quando deixado sob desenvolvimento livre; assim, Talismã obteve uma produção decenal média de 8,12 kg.planta ${ }^{-1}$ (BARBOSA et al., 1999). Nota-se, nesse caso, uma redução de cerca de $40 \%$ na produção, em ciclos cuja poda drástica já havia sido realizada.

Dada a flexibilidade que possuem os pessegueiros em responder aos diferentes tipos de poda e condução de planta, várias pesquisas vêm sendo realizadas no sentido de simplificar ou mecanizar seu cultivo. $\mathrm{O}$ manejo mais comum, utilizado na Europa, relacionase aos sistemas "Fusetto", "Tatura", "Palmetta" e "Hedgerow", em que se aplica a poda manual ou mecanizada de encurtamento dos ramos em pré e pós-colheita, para controle da vegetação e melhoria da qualidade do fruto (BARGIONI et al., 1983; MARINI, 1985; Loreti et al., 1991; LoRETI e PisAnI, 1990; CARUSO et al., 1997). Nas condições climáticas paulistas, uma poda de encurtamento acentuado dos ramos, em póscolheita, poderia ser viável com vantagem em relação ao método de decepamento do tronco do pessegueiro. Conjecturou-se, então, que os ramos de produção, se encurtados bem próximos à sua base, reconstituiriam idealmente a copa evitando a frutificação alternada ou reduzida, decorrentes de ciclos pós-podas drásticas. Objetivou-se, neste trabalho, comparar o desenvolvimento de catorze cultivares e seleções de pessegueiro e nectarineira, em pomar compacto, com poda de encurtamento dos ramos, em pós-colheita.

\section{MATERIAL E MÉTODOS}

Utilizaram-se na experimentação catorze cultivares e seleções de pessegueiros e nectarineiras cultivados no sistema de pomar compacto, na Estação Experimental de Agronomia de Monte Alegre do Sul, do Instituto Agronômico (22 $41^{\prime} \mathrm{S}$; $46^{\circ} 43^{\prime} \mathrm{W}$ e $40 \mathrm{HF}-$ 
7)(Pedro Junior et al., 1979). Os cultivares e seleções foram: Aurora-1, Régis, Talismã, Flordaprince, Jóia-1, Tropical, Delicioso Precoce, IAC 680-13, IAC 680-178 e IAC 2380-55 (pêssegos); Centenária, Josefina, Rubro-sol (Sunred) e IAC N 2680-91 (nectarinas) obtidos ou introduzidos pelo programa de melhoramento genético do IAC (OJIMA et al., 1993). São pêssegos e nectarinas que apresentam ciclos de maturação classificados como bem precoce, precoce e mediano (BARBOSA et al., 1990b).

O lote experimental foi conduzido no espaçamento de $4 \mathrm{~m} \times 1,5 \mathrm{~m}$ (1.667 plantas.ha $\left.{ }^{-1}\right) \mathrm{com}$ cinco plantas por cultivar e seleção, as quais foram formadas em recipientes de plástico, utilizando-se o pessegueiro Okinawa como porta-enxerto. Os pessegueiros e nectarineiras com 12, 13 e 14 anos em 1996, 1997 e 1998, respectivamente, conduzidos com cinco pernadas, foram submetidos anualmente à poda de encurtamento dos ramos. Efetuou-se o encurtamento dos ramos produtivos no final de novembro, de maneira que os mesmos permanecessem com 20 $30 \mathrm{~cm}$ de comprimento a partir de sua inserção. Segundo BARBOSA et al. (1999), essas plantas foram, nos dez primeiros anos, conduzidas sob poda drástica bienal da copa pós-colheita As plantas receberam tratos culturais rotineiros, como: caiação do tronco, capina, tratamento fitossanitário das folhas e frutos, irrigação no período reprodutivo, correção de acidez do solo, adubação química e quebra-de-endodormência. Para facilitar o manejo, os pessegueiros e nectarineiras foram rebaixados a cerca de $2,5 \mathrm{~m}$ de altura, no inverno; nessa ocasião fez-se a retirada dos ramos secos e doentes. No raleio foram deixados dois e três frutos por ramo médio e vigoroso respectivamente. Durante os dois anos de pesquisa efetuou-se, por safra, o controle da produção de frutos (número e massa) colhidos em cinco plantas, por cultivar e seleção.

Em meados de novembro de 1997 e 1998, foram determinados a área de secção transversal do tronco e o volume da copa. A área de secção transversal do tronco $\left(\mathrm{cm}^{2}\right)$ foi obtida convertendo-se a medida do diâmetro do tronco $(\mathrm{cm})$, determinada com um paquímetro colocado a cerca de $20 \mathrm{~cm}$ do solo, com a tabela apresentada por WeSTWOOD (1978). O volume da copa foi calculado mediante as medidas de altura e largura, empregando-se a fórmula: $4 / 3 a b^{2}$, em que " $a$ " refere-se a do eixo maior, e " $b$ ", a $1 / 2$ do eixo menor (WestWOOD, 1978). A produção por $\mathrm{cm}^{2}$ de tronco foi estimada dividindo-se o número e a massa dos frutos pela área de secção tranversal de tronco de cada planta. Efetuou-se a análise da variância, e as médias foram comparadas pelo teste de Tukey (5\%). Calculou-se, também, o coeficiente de correlação sim- ples para as principais características fisiológicas de produção.

\section{RESULTADOS E DISCUSSÃO}

Apenas dez dias após a poda de encurtamento, iniciou-se intensa brotação das gemas, principalmente, na porção apical dos ramos. O desenvolvimento das brotações persistiu até o final de março, em Monte Alegre do Sul, com dias quentes e longos. As gemas axilares das novas brotações desenvolveram-se a partir do final de janeiro e, em fins de fevereiro, apresentavam-se com o desenvolvimento fenológico máximo. Em meados de abril, cinco meses após a poda, verificou-se menor desenvolvimento das plantas. Isso ocorreu devido à diminuição paulatina do fotoperíodo, que acontece a partir do final de dezembro. Ao observar os valores da radiação solar verifica-se, no Estado de São Paulo, que há um sensível aumento, de setembro a janeiro, devido ao alongamento dos dias; após esse período, o dia diminui gradativamente até julho, afetando e reduzindo o desenvolvimento vegetativo das plantas brevediurnas, como o pessegueiro (FRANCIS, 1972; PEDRO JUNIOR et al., 1989). Assim, de maneira geral, quanto mais cedo se proceder à poda de encurtamento dos ramos, maiores deverão ser os efeitos benéficos no desenvolvimento das gemas florais. No presente experimento, optou-se por retardar em cerca de vinte dias a poda dos cultivares bem precoces e precoces, evitando-se a defasagem de crescimento em relação aos medianos, cujas colheitas ocorrem em meados de novembro. Isso, porém, não afetou a formação de gemas florais, floração e frutificação efetiva nas brotações novas dos pessegueiros e nectarineiras pesquisados.

Os pessegueiros Aurora-1, IAC 680-13, Régis, Talismã e IAC 680-178 apresentaram melhor produtividade e potencial para cultivo em elevada densidade de plantio, com poda de encurtamento dos ramos após a colheita (Quadros 1 e 2). Verificou-se que esse tipo de poda evitou a alternância de produção, visto que a frutificação dos cultivares e a das seleções foram similares nos dois anos consecutivos da experimentação. Em cultivos perenes como o do pessegueiro, pequenas diferenças produtivas verificadas de um ano para outro podem ser consideradas normais, à exceção de IAC 2380-55 que, devido à causa não identificada, diminuiu a produção em cerca de $50 \%$, no segundo ano do experimento.

Vale salientar que esses cinco cultivares e seleções mais produtivos apresentam polpa amarela e firme e linhagem genealógica similar (BARBOSA et al., 1997). Além disso, essas seleções IAC têm em comum a 
W. BARBOSA et al.

Quadro 1. Desenvolvimento, produção e índices fisiológicos de 14 pessegueiros e nectarineiras cultivados em pomar compacto (4 m x 1,5 m), sob poda de encurtamento dos ramos, pós-colheita, na Estação Experimental de Agronomia de Monte Alegre do Sul (SP). Valores médios obtidos de cinco plantas, por cultivar e seleção, no ano agrícola de 1997. Instituto Agronômico, 1999

\begin{tabular}{|c|c|c|c|c|c|c|c|}
\hline \multirow{3}{*}{ Material } & \multicolumn{7}{|c|}{ Características fisiológicas } \\
\hline & \multirow{2}{*}{$\begin{array}{c}\text { Área } \\
\text { transversal } \\
\text { do tronco }\end{array}$} & \multirow{2}{*}{$\begin{array}{c}\text { Volume da } \\
\text { copa }\end{array}$} & \multicolumn{3}{|c|}{ Produção de frutos por planta } & \multicolumn{2}{|c|}{ Índices fisiológicos } \\
\hline & & & Fruto & Massa & Massa média & $\begin{array}{c}\text { Frutos } / \mathrm{cm}^{2} \\
\text { de tronco }\end{array}$ & $\begin{array}{c}\text { Massa } / \mathrm{cm}^{2} \\
\text { de tronco }\end{array}$ \\
\hline & $\mathrm{cm}^{2}$ & $\mathrm{~m}^{3}$ & $n^{\circ}$ & $\mathrm{kg}$ & $\mathrm{g}$ & $\mathrm{n}^{\circ} \cdot \mathrm{cm}^{-2}$ & g.cm ${ }^{-2}$ \\
\hline Aurora-1 & $150,6 \operatorname{fgh}^{(1)}$ & $9,25 \mathrm{abc}$ & $149 a$ & $13,34 a$ & $89,7 \mathrm{~b}$ & $0,99 a$ & $88,6 a$ \\
\hline IAC 680-13 & $280,0 \mathrm{a}$ & $8,92 \mathrm{bcd}$ & $143 a$ & $12,46 \mathrm{ab}$ & $87,5 b$ & $0,51 \mathrm{ef}$ & 44,5 de \\
\hline Régis & $162,2 \mathrm{efg}$ & $7,76 \mathrm{ef}$ & $135 \mathrm{ab}$ & $11,59 b$ & $86,4 \mathrm{bc}$ & $0,83 \mathrm{bc}$ & $71,5 b$ \\
\hline Talismã & $153,6 f g h$ & $9,81 \mathrm{ab}$ & $137 \mathrm{a}$ & $12,45 \mathrm{ab}$ & $91,0 b$ & $0,89 \mathrm{ab}$ & $81,1 \mathrm{ab}$ \\
\hline IAC 680-178 & $164,8 \mathrm{efg}$ & 8,09def & $114 \mathrm{bc}$ & $9,27 \mathrm{c}$ & $81,3 \mathrm{~cd}$ & $0,69 \mathrm{~cd}$ & $56,2 \mathrm{c}$ \\
\hline Flordaprince & $221,6 \mathrm{bc}$ & 8,74 bcde & $95 \mathrm{~cd}$ & $8,66 \mathrm{~cd}$ & $90,8 b$ & $0,43 \mathrm{fg}$ & $39,1 \mathrm{ef}$ \\
\hline Jóia-1 & $137,6 \mathrm{gh}$ & 8,10def & $89 d$ & 7,20de & 80,8 cde & $0,65 \mathrm{de}$ & $52,4 \mathrm{~cd}$ \\
\hline IAC 2380-55 & $168,0 \mathrm{ef}$ & $7,79 \mathrm{ef}$ & $150 \mathrm{a}$ & $11,90 \mathrm{ab}$ & $79,7 \mathrm{de}$ & $0,89 \mathrm{ab}$ & $70,9 b$ \\
\hline Tropical & $243,8 b$ & 8,83 bcde & $95 \mathrm{~cd}$ & 7,18de & $75,3 \mathrm{ef}$ & 0,39 fgh & $29,6 \mathrm{fg}$ \\
\hline Delic. Precoce & $126,6 \mathrm{~h}$ & $7,06 f$ & $57 e$ & $6,36 \mathrm{ef}$ & $111,4^{\mathrm{a}}$ & $0,45 \mathrm{fg}$ & 50,3 cde \\
\hline IAC N 2680-91 & $151,4 \mathrm{fgh}$ & $7,01 \mathrm{f}$ & $56 e$ & $3,91 \mathrm{~g}$ & $69,4 \mathrm{f}$ & 0,39 fgh & $27,1 \mathrm{~g}$ \\
\hline Centenária & $211,4 \mathrm{~cd}$ & $10,11 a$ & $66 \mathrm{e}$ & $4,65 \mathrm{fg}$ & $70,3 \mathrm{f}$ & $0,31 \mathrm{gh}$ & $22,0 \mathrm{~g}$ \\
\hline Josefina & $218,6 \mathrm{bcd}$ & 8,79 bcde & $60 \mathrm{e}$ & $4,76 \mathrm{fg}$ & $78,9 \mathrm{de}$ & $0,28 \mathrm{~h}$ & $21,8 \mathrm{~g}$ \\
\hline Rubro-sol & $190,8 \mathrm{de}$ & $8,27 \mathrm{cde}$ & $59 e$ & $4,08 \mathrm{~g}$ & $69,5 f$ & $0,31 \mathrm{gh}$ & $21,4 \mathrm{~g}$ \\
\hline F (Trat) & $57,43^{* *}$ & $16,39^{* *}$ & $68,33^{* *}$ & $97,63^{* *}$ & $86,89^{* *}$ & $69,38^{* *}$ & $99,59 * *$ \\
\hline CV (\%) & 7,14 & 6,00 & 9,94 & 9,25 & 3,24 & 11,60 & 10,64 \\
\hline Dms 5\% & 29,30 & 1,13 & 22,22 & 1,73 & 5,99 & 0,15 & 11,45 \\
\hline
\end{tabular}

(1)Valores na coluna, seguidos pela mesma letra, não diferem entre si, pelo teste de Tukey a 5\%.

**Significativo a $1 \%$.

descendência em $1 .^{\mathrm{a}}, 2 .^{\mathrm{a}}$ e $3 .^{\mathrm{a}}$ gerações do Rei da Conserva - pêssego antigo, rústico e do tipo conserva. A produtividade média bienal desses pessegueiros situou-se em patamares de 16,8 a 23,1 t.ha ${ }^{-1}$, considerados adequados para pomares compactos, nas condições locais de inverno brando. BARGIONI et al. (1985), ao pesquisarem pessegueiros em pomar compacto na Itália, relatam que um rendimento acima de 15 t.ha $^{-1}$ já seria satisfatório para espaçamentos de $4 \mathrm{~m} \times 1 \mathrm{~m}$, $4 \mathrm{~m} \times 1,5 \mathrm{~m}$ e $4 \mathrm{~m} \times 2 \mathrm{~m}$. Tanto esses autores como RECUPERO et al. (1985) concluíram que densidades populacionais próximas a 2.000 plantas.ha ${ }^{-1}$ podem ser mais viáveis para se conciliar alta produtivididade e melhor qualidade do fruto, além das facilidades nos tratos culturais. Nesse aspecto, CARUSO et al. (1997), pesquisando pessegueiros precoces e de menor exigência em clima frio, relataram produções entre 9 e $13 \mathrm{~kg} \cdot$ planta $^{-1}$ em densidade populacional de 2.000 plantas.ha ${ }^{-1}$, sob os sistemas "Fusetto" $\mathrm{e}^{\text {"Tatura }}$ livre", com poda de encurtamento dos ramos pós-co- lheita. Quanto aos pessegueiros 'Flordaprince', com três anos, obtiveram índices de 11 kg, 115,5 g e 130 g, respectivamente, para produção por planta, massa média dos frutos e massa por $\mathrm{cm}^{2}$ de área transversal de tronco. Neste trabalho, as relações médias entre produção e área transversal de tronco foram: 48,3 e 45,8 g.cm ${ }^{-2}$, em 1997 e 1998 respectivamente. Tais valores, relativamente baixos, foram obtidos devido à grande área transversal do tronco dos pessegueiros adultos. Na idade de 13 e 14 anos seus troncos, com diâmetros maiores, sustentam copas idênticas às da época em que eram mais jovens, com índices de área transversal significativamente menores. Assim, em pessegueiro conduzido sob pomar compacto, com poda drástica da copa, quanto maior a área transversal de tronco, menor será a relação de fruto e massa por $\mathrm{cm}^{2}$ de tronco. As correlações da área de secção transversal de tronco vs. o número total de frutos e a massa total de frutos não apresentaram relevância estatística. 
Quadro 2. Desenvolvimento e produção de frutos de cultivares e seleções de pessegueiros e nectarineiras em pomar compacto (4 m x 1,5 m), sob poda de encurtamento dos ramos, pós-colheita, na Estação Experimental de Agronomia de Monte Alegre do Sul (SP). Valores médios obtidos em cinco plantas, por cultivar e seleção, no ano agrícola de 1998. Instituto Agronômico, 1999

\begin{tabular}{|c|c|c|c|c|c|c|c|}
\hline \multirow{3}{*}{ Material } & \multicolumn{7}{|c|}{ Características fisiológicas } \\
\hline & \multirow{2}{*}{$\begin{array}{c}\text { Área } \\
\text { transversal } \\
\text { do tronco }\end{array}$} & \multirow{2}{*}{$\begin{array}{c}\text { Volume da } \\
\text { copa }\end{array}$} & \multicolumn{3}{|c|}{ Produção de frutos por planta } & \multicolumn{2}{|c|}{ Índices fisiológicos } \\
\hline & & & Fruto & Massa & Massa média & $\begin{array}{c}\text { Frutos } / \mathrm{cm}^{2} \\
\text { de tronco }\end{array}$ & $\begin{array}{c}\text { Massa } / \mathrm{cm}^{2} \\
\text { de tronco }\end{array}$ \\
\hline & $\mathrm{cm}^{2}$ & $\mathrm{~m}^{3}$ & $n^{\circ}$ & $\mathrm{kg}$ & $\mathrm{g}$ & $\mathrm{n}^{\mathrm{o}} \cdot \mathrm{cm}^{-2}$ & g.cm ${ }^{-2}$ \\
\hline Aurora-1 & $156,8 \mathrm{fg}^{(1)}$ & $9,32 \mathrm{bcd}$ & $173 a b$ & $14,57 \mathrm{a}$ & $84,2 \mathrm{cde}$ & $1,11 \mathrm{a}$ & $93,1 \mathrm{a}$ \\
\hline IAC 680-13 & $289,4^{\mathrm{a}}$ & $9,17 \mathrm{bcd}$ & $141 b c$ & $12,36 \mathrm{abc}$ & $87,7 \mathrm{bcd}$ & $0,49 d$ & $42,7 \mathrm{c}$ \\
\hline Régis & $171,4 \mathrm{ef}$ & $7,83 \mathrm{ef}$ & $178 \mathrm{a}$ & $12,99 \mathrm{ab}$ & $72,9 \mathrm{fg}$ & $1,04 \mathrm{ab}$ & $75,9 \mathrm{ab}$ \\
\hline Talismã & $165,6 f$ & $10,37 \mathrm{ab}$ & $120 \mathrm{~cd}$ & $11,49 \mathrm{bcd}$ & $96,5 \mathrm{ab}$ & $0,73 c$ & $69,8 b$ \\
\hline IAC 680-178 & $175,0 \mathrm{ef}$ & $8,82 \mathrm{cde}$ & $146 \mathrm{abc}$ & $10,93 \mathrm{bcd}$ & $74,1 \mathrm{efg}$ & $0,84 b c$ & $62,8 b$ \\
\hline Flordaprince & $231,0 \mathrm{bc}$ & $9,33 \mathrm{bcd}$ & 96de & 8,87de & $92,9 b c$ & 0,42 de & $38,5 \mathrm{~cd}$ \\
\hline Jóia-1 & $145,0 \mathrm{fg}$ & 8,30def & $144 \mathrm{abc}$ & $9,87 \mathrm{~cd}$ & $68,8 \mathrm{fg}$ & $0,99 \mathrm{ab}$ & $68,1 b$ \\
\hline IAC 2380-55 & $176,4 \mathrm{ef}$ & $8,79 \mathrm{cde}$ & $73 \mathrm{efg}$ & $5,73 f$ & 78,2 def & 0,42 de & $32,5 \mathrm{cde}$ \\
\hline Tropical & $258,0^{a} b$ & $9,67 \mathrm{abc}$ & $84 \mathrm{ef}$ & $6,39 \mathrm{ef}$ & $76,2 \mathrm{efg}$ & $0,33 \mathrm{de}$ & $24,8 \mathrm{de}$ \\
\hline Delici. Precoce & $132,4 \mathrm{~g}$ & $7,24 \mathrm{f}$ & $54 \mathrm{fg}$ & $5,64 f$ & $104,7 a$ & $0,41 \mathrm{de}$ & $42,4 \mathrm{c}$ \\
\hline IAC N 2680-91 & $159,8 \mathrm{fg}$ & $7,20 f$ & $67 \mathrm{efg}$ & $4,64 \mathrm{f}$ & $69,7 \mathrm{fg}$ & 0,43de & 29,9 cde \\
\hline Josefina & $225,8 \mathrm{~cd}$ & $9,08 \mathrm{cde}$ & $64 \mathrm{efg}$ & $4,21 \mathrm{f}$ & $65,9 \mathrm{~g}$ & $0,28 \mathrm{de}$ & $18,7 \mathrm{e}$ \\
\hline Centenária & $222,4 \mathrm{~cd}$ & $10,85 a$ & $71 \mathrm{efg}$ & $5,46 \mathrm{f}$ & 77,4 def & $0,32 \mathrm{de}$ & $24,6 \mathrm{de}$ \\
\hline Rubro-sol & 198,8 de & 8,61cde & $49 \mathrm{~g}$ & $3,61 \mathrm{f}$ & 73,9efg & $0,25 \mathrm{e}$ & $18,2 \mathrm{e}$ \\
\hline F (Trat) & $50,47^{* *}$ & $16,40^{* *}$ & $40,58^{* *}$ & $41,02^{* *}$ & $29,86^{* *}$ & $47,56^{* *}$ & $45,80^{* *}$ \\
\hline CV (\%) & 7,44 & 6,51 & 15,15 & 15,37 & 5,84 & 17,10 & 17,18 \\
\hline Dms 5\% & 32,03 & 1,29 & 35,19 & 2,85 & 10,43 & 0,22 & 17,54 \\
\hline
\end{tabular}

${ }^{(1)}$ Valores na coluna, seguidos pela mesma letra, não diferem entre si, pelo teste de Tukey a 5\%.

**Significativo a $1 \%$.

Quando se consideram as plantas manejadas tradicionalmente, verificam-se valores como $95 \mathrm{~g} . \mathrm{cm}^{-2}$ (SCORZA et al., 1986) e, aproximadamente, cinco frutos por $\mathrm{cm}^{2}$ de área de secção transversal de tronco (FELICIANO et al., 1984). Esse padrão de cinco frutos por $\mathrm{cm}^{2}$ não pode ser transportado para o sistema de pomar compacto, pois levaria a um número excessivamente alto de pêssegos e nectarinas - naturalmente pequenos - por planta. Com o avanço da idade, do crescimento lento e contínuo do tronco e da manutenção constante do volume da copa, a citada relação causaria diminuição drástica no tamanho dos frutos. As altas correlações obtidas entre a massa média e o número de frutos $(\mathrm{r}=86)$ e entre a massa média de frutos e a área de secção transversal de tronco $(r=90)$, comprovam estatisticamente esse fato.

Os pessegueiros com produção intermediária, como Delicioso Precoce, Tropical, Jóia-1, Flordaprince e IAC 2380-55 obtiveram valores médios entre $6,0 \mathrm{~kg}$ e $8,8 \mathrm{~kg}$ de frutos por planta, correspondentes a 10,0 e 14,7 tha ${ }^{-1}$ respectivamente. Esses cultivares melhoraram a produtividade e a massa média dos frutos, quando comparados aos tratamentos de podas drásticas bienais da copa (BARBOSA et al., 1999). Apesar do aumento de massa média dos frutos, os cultivares e as seleções apresentaram, ainda, pêssegos e nectarinas entre $10 \%$ e $30 \%$ inferiores ao sistema convencional. Esses resultados foram também relatados por outros autores, ao pesquisarem as altas densidades de plantio de pessegueiro e nectarineira (EREZ, 1976; CAMPO DALL'ORTO et al.., 1984; ReCUPERO et al., 1985; Bellini et al., 1985; Scarpare Filho et al., 1999). Os maiores frutos foram observados em Delicioso Precoce, com massa média de 111,4 g, em 1997, consideravelmente acima dos demais cultivares e seleções (Quadro 1). Os menores frutos foram produzidos pelas nectarineiras, principalmente em IAC N 2680-91 e Rubro-sol, com massa média inferior a 70 g. Além 
disso, as nectarinas apresentaram também as menores produções, sempre abaixo de 5,5 kg.planta ${ }^{-1}$.

\section{CONCLUSÕES}

1. Os cinco cultivares e seleções de maior produtividade: Aurora-1, IAC 680-13, Régis, Talismã e IAC 680-178 revelaram-se como os mais adaptados ao sistema de pomar compacto sob poda de encurtamento dos ramos, em pós-colheita. Esses pessegueiros produziram, em média bienal, entre 16,8 e 23,1 t.ha ${ }^{-1}$.

2. O desbaste de frutos com base no número de ramos, ou seja, no volume da copa, apresenta-se como a forma mais indicada para o sistema adensado de plantio de pessegueiros e nectarineiras, qualquer que seja o tipo de poda adotado.

3. As nectarineiras apresentaram as mais baixas produções de frutos, por planta, demostrando menor adaptabilidade ao sistema pesquisado.

4. De modo geral, os cultivares e seleções de pessegueiro e nectarineira pesquisados não apresentaram alternância de produção, quando conduzidos em pomar compacto sob poda de encurtamento dos ramos, em pós-colheita.

5. Pessegueiros e nectarineiras, como IAC 670-13, Tropical, Josefina e Rubro-sol, conduzidos em pomar compacto, com podas sucessivas da copa, demostraram que, quanto maior a área transversal do tronco, menor o índice de frutos e de massa por $\mathrm{cm}^{2}$ de tronco.

\section{AGRADECIMENTOS}

Ao pessoal de apoio, Lázaro de Godoi, Marcilene de Moraes, Antonio Carlos de Carvalho, Meire Correia da Silva Ferrari e Valdemir Álvares, pelos tratos culturais das plantas e pelo levantamento de dados dos experimentos, e ao pesquisador científico Nilberto Bernardo Soares, pela revisão do texto.

\section{REFERÊNCIAS BIBLIOGRÁFICAS}

BARBOSA, W. Desenvolvimento vegetativo e reprodutivo do pessegueiro em pomar compacto sob poda drástica anual. Piracicaba, 1989. 154p. Dissertação (Mestrado em Agronomia) - ESALQ-USP, 1989.

BARBOSA, W.; CAMPO-DALL'ORTO, F.A.; OJIMA, M. O pessegueiro no sistema de pomar compacto: I. Conjecturas, experimentação e prática. O Agronômico. Campinas, v.41, n.1, p.26-39, 1989.

BARBOSA, W.; CAMPO-DALL'ORTO, F.A.; OJIMA, M.; LOVATE, A.A.; SANTOS, R.R. O pessegueiro no sistema de pomar compacto: VII. Comportamento de novas seleções IAC sob poda drástica bienal. Scientia Agricola, Piracicaba, v.51, n.1, p.94-98, 1994.
BARBOSA, W.; CAMPO-DALL'ORTO, F.A.; OJIMA, M.; SAMPAIO, V.R. O pessegueiro no sistema de pomar compacto: III. Épocas de poda drástica na diferenciação floral. Bragantia, Campinas, v.49, n.1, p.147-155, 1990a.

BARBOSA, W.; CAMPO-DALL'ORTO, F.A.; OJIMA, M.; SANTOS, R R. O pessegueiro no sistema de pomar compacto: VII. Caracterização de cultivares e seleções para diferentes densidades populacionais. Scientia Agricola, Piracicaba, v.55, n.2, p.191-195, 1998.

BARBOSA, W.; OJIMA, M.; CAMP-DALL'ORTO, F A.; MARTINS, F.P. Época e ciclo de maturação de pêssegos e nectarinas no Estado de São Paulo. Bragantia, Campinas, v.49, n.2, p 221-226, 1990 b.

BARBOSA, W.; OJIMA, M.; CAMPO-DALL'ORTO, F.A.; RIGITANO, O.; MARTINS, F.P.; SANTOS, R.R.; CASTRO, J.L. Melhoramento do pessegueiro para regiões de clima subtropical-temperado: realizações do Instituto Agronômico no período de 1950/1990. Campinas: Instituto Agronômico, 1997. 22p. (Documentos IAC, 52)

BARBOSA, W.; OJIMA, M.; CAMPO-DALL'ORTO, F.A.; SAMPAIO, V.R.; MARTINS, F.P.; IGUE, T. O pessegueiro no sistema de pomar compacto: II. Influência das épocas de poda drástica no desenvolvimento das plantas. Campinas: Instituto Agronômico, 1991. 20 p. (Boletim Científico, 22)

BARBOSA, W.; CAMPO-DALL'ORTO, F.A.; OJIMA, M.; SANTOS, R R.; KALIL, G.P.C.; FAHL, J.I.; CARELLI, M.L.C. O pessegueiro em pomar compacto: IX. Dez anos de produção de cultivares sob poda drástica bienal. Pesquisa Agropecuária Brasileira, Brasília, v.34, n.1, p.69-76, 1999.

BARGIONI, G.; LORETI, F.; PISANI, P.L. Performance of peach and nectarine in high density system in Italy. HortScience, Alexandria, v.18, n.2, p.143-146, 1983.

BARGIONI, G.; LORETI, F.; PISANI, P.L. Ten years of research on peach and nectarine in a hight density system in the Verona area. Acta Horticulturae, Wageningen, v.173, p.229-309, 1985.

BELLINI, E.; CIMATO, A ; MARIOTTI, P. Six years observations on a meadow orchard of self-rooted nectarines in protected culture. Acta Horticulturae, Wageningen, v.173, p.329-338, , 1985.

CAMPO-DALL'ORTO, F.A.; OJIMA, M.; BARBOSA, W.; TOMBOLATO, A.F.C.; RIGITANO, O., ALVES, S. Cultivo de seleções de pessegueiros precoces no sistema de pomar compacto com poda drástica anual de renovação da copa. Pesquisa Agropecuária Brasileira, Brasília, v.19, n.6, p.719-727, 1984.

CARUSO, T.; GIOVANNINI, D.; MARRA, F.P.; SOTTILE, F. Two new systems for early ripening peaches (Prunus persica L. Batsch): Yield and fruit quality in four lowchilling cultivars. Journal of Horticultural Science, v.72, n.6, p.873-883, 1997. 
COSTON, D.C.; SIMS JUNIOR, E.T.; WILLIANSON, R.E. Productivity of peach meadow orchards. Acta Horticulturae, Wageningen, v.173, p.323-328, 1985.

EREZ, A. Meadow orchard for the peach. Scientia Horticulturae, Amsterdam, v.5, p.43-48, 1976.

EREZ, A. Peach meadow orchards. Acta Horticulturae, Wageningen, v.173,, p.405-411, 1985.

FELICIANO, A.J.; NAKASU, B.H.; SACHS, S. Raleio. In: EMBRAPA/Centro Nacional de Pesquisa de Fruteiras de Clima Temperado. A cultura do pessegueiro. Pelotas, 1984. 156p. (Circular Técnica, 10)

FRANCIS, C.A. Natural daylenghts for photoperiod sensitive plants. Cali, Colombia: CIAT, 1972. 32p. (Technical Bulletin, 2)

GUERRIERO, B.; LORETI, F.; NATALI, S. Eight years of observations on a peach double-row planted orchard. Acta Horticulturae, Wageningen, v.114, p.362-363, 1980.

LORETI, F.; PISANI, P L. Elevate densità di piantagione e relative forme de allevamento. Atti Convegno "La potatura degli alberi da frutto negli anni 90", Verona, v.27, p.38-68, 1990.

LORETI, E.; MASSAI, B.; MORINI, S. Effect of training system, planting density and rootstock on growth and quality of peach shoots. Advances in Horticultural Science, New York, v.3, p.45-47, 1991.

MARINI, R.P. Vegetative growth, yield, and fruit quality of peach as influenced by dormant prunning, summer pruning, and summer topping. Journal of the American for Society Horticultural Science, New York, v.110, n.2, p.133-139, 1985.
OJIMA, M.; CAMPO-DALL'ORTO, F.A.; BARBOSA, W.; RIGITANO, O. Frutas de Clima Temperado. In: FURLANI, A. M. C.; VIÉGAS, G.P.(Eds). O Melhoramento de Plantas no Instituto Agronômico. Campinas: Instituto Agronômico, 1993. 524p.

PEDRO JUNIOR, M.J.; ALFONSI, R R.; CAMARGO, M.B P.; CHIAVEGATTO, O.M.D.P.; ORTOLANI, A.A.; BRUNINI, O. Disponibilidade de radiação solar para o Estado de São Paulo. Campinas: Instituto Agronômico, 1989. 12p. (Boletim Técnico, 129)

PEDRO JUNIOR, M.J.; ORTOLANI, A.A.; RIGITANO, O.; ALFONSI, R R.; PINTO, H.S.; BRUNINI, O. Estimativa de horas de frio abaixo de 7 e $13^{\circ} \mathrm{C}$ para regionalização da fruticultura de clima temperado no Estado de São Paulo. Bragantia, Campinas, v.38, p.123-130, 1979.

RECUPERO, S.; MONASTRA, F.; DAMIANO, C. Study on planting densities of peach and nectarine cultivars on different rootstocks. Acta Horticulturae, Wageningen, v.173, n.3, p.311-322, 1985.

SCARPARE FILHO, J.A.; KLUGE, R.A.; JACOMINO, A.P.; TESSARIOLI NETO, J. Comparação entre dois sistemas de condução de pessegueiros em pomar compacto. Revista Brasileira de Fruticultura, Jaboticabal, v.21, n.1, p.92-94, 1999.

SCORZA, R.; ZAILONG, L.; LIGHTNER, G.W.; GILREATH, L.E. Dry matter distribution and responses to pruning within a population of standard, semidwarf compact, and dwarf peach seedlings. Journal of the American Society for Horticultural Science, New York, v.111, n.4, p.541-545, 1986.

WESTWOOD, M. N. Temperate zone pomology. San Francisco: W.H.Freeman, 1978. 428p. 